Original paper

\title{
Optimizing beam models for dosimetric accuracy over a wide range of treatments
}

\author{
Josephine Chen*, Olivier Morin, Brandon Weethee, Angelica Perez-Andujar, Justin Phillips, \\ Mareike Held, Vasant Kearney, Dae Yup Han, Joey Cheung, Cynthia Chuang, Gilmer Valdes, \\ Atchar Sudhyadhom, Timothy Solberg
}

Department of Radiation Oncology, University of California San Francisco, 1600 Divisadero Street, Suite H1031, San Francisco, CA 94115, United States

\section{A R T I C L E I N F O}

\section{Keywords:}

External beam

Treatment planning

Radiotherapy

\begin{abstract}
A B S T R A C T
This work presents a systematic approach for testing a dose calculation algorithm over a variety of conditions designed to span the possible range of clinical treatment plans. Using this method, a TrueBeam STx machine with high definition multi-leaf collimators (MLCs) was commissioned in the RayStation treatment planning system (TPS). The initial model parameters values were determined by comparing TPS calculations with standard measured depth dose and profile curves. The MLC leaf offset calibration was determined by comparing measured and calculated field edges utilizing a wide range of MLC retracted and over-travel positions. The radial fluence was adjusted using profiles through both the center and corners of the largest field size, and through measurements of small fields that were located at highly off-axis positions. The flattening filter source was adjusted to improve the TPS agreement for the output of MLC-defined fields with much larger jaw openings. The MLC leaf transmission and leaf end parameters were adjusted to optimize the TPS agreement for highly modulated intensity-modulated radiotherapy (IMRT) plans. The final model was validated for simple open fields, multiple field configurations, the TG 119 C-shape target test, and a battery of clinical IMRT and volumetric-modulated arc therapy (VMAT) plans. The commissioning process detected potential dosimetric errors of over $10 \%$ and resulted in a final model that provided in general 3\% dosimetric accuracy. This study demonstrates the importance of using a variety of conditions to adjust a beam model and provides an effective framework for achieving high dosimetric accuracy.
\end{abstract}

\section{Introduction}

Performing the dosimetric commissioning of an external beam treatment planning system (TPS) is an important medical physics task that impacts the accuracy for all plans created using the commissioned beam models. Both the AAPM and the IAEA have devoted publications to providing guidance on the commissioning process [1-5]. Identifying beam model parameters that provide accurate dose calculations over a wide range of clinical treatment types can be challenging. There have been several publications that have described situations in which a beam model was tested and released for clinical use but as the usage of the planning system expanded to more complex treatment sites, the plans began to fail the institution's measurement-based plan quality assurance testing [6,7]. This prompted the institutions to perform additional measurements to further refine their beam models. Others have noted that different optimal values for model parameters can be found for different plans [8]. Recently, the IROC-Houston analyzed 259 head and neck phantom irradiations and found that $20 \%$ of institutions demonstrated errors in their treatment planning system calculations [9].

The goal of this work is to present a thorough and systematic approach to testing a photon beam model over a variety of conditions designed to span the possible range of clinical treatment plans and delivery techniques. In this work, a modern linear accelerator with a high definition multi-leaf collimator (MLC) was commissioned in the RayStation treatment planning system. Although not presented, a similar approach has been used to commission beam models in the Pinnacle treatment planning system. A series of tests were devised and used to guide the modification of beam model parameter values beyond the initial values found by comparing the imported beam data with the TPS calculations. The dosimetric accuracy of the beam models was verified by testing the model using a battery of clinical cases in a variety of quality assurance (QA) phantoms. Specific parameters that were

\footnotetext{
* Corresponding author.

E-mail address: josephine.chen@kp.org (J. Chen).
} 
adjusted in the RayStation beam model are presented, but the general process can be applied to any treatment planning system.

\section{Materials and methods}

\subsection{Linear accelerator and treatment planning system}

The linear accelerator modeled was a TrueBeam STx machine with a high definition MLC (Varian Medical Systems, Palo Alto, CA). The high definition MLC consists of 60 leaf pairs that span the central $22 \mathrm{~cm}$ length of the field in the gun-target direction. The inner 32 leaf pairs project a width of $0.25 \mathrm{~cm}$ at isocenter while the outer leaves project a width of $0.5 \mathrm{~cm}$. The leaves move linearly, interdigitate, and have rounded leaf tips. Five beam lines were modeled: three flattened beams $(6,10,15 \mathrm{MV})$ and two flattening filter free beams (6 FFF, $10 \mathrm{FFF})$. The standard beam data for open, jaw-defined fields was acquired using TG 106 recommendations [10] and imported into the RayStation treatment planning system, version 5.0.2 (Raysearch Laboratories AB, Stockholm, Sweden). The RayStation photon dose calculation algorithm employs a semi-empirical model of the photon/electron fluence emanating from the accelerator head [11] and a collapsed cone convolution/superposition dose calculation engine to calculate the deposited dose $[12,13]$. The fluence model includes parameters to represent the energy spectrum of the photons and contamination electrons, the off-axis softening of the spectrum, radial changes in the fluence magnitude, and a flattening filter scattering source. The transmission through the $\mathrm{X}$ jaws is modeled by a transmission factor, the transmission through the $\mathrm{Y}$ jaws is assumed to be zero, and the transmission through the MLCs is modeled by a transmission factor, a tongue and groove width, and a leaf tip width. As has previously been described [14], the distal end of the MLC leaf is modeled as a region of increased transmission, defined as the squared-root of the MLC leaf transmission. The length of this region is defined by the leaf tip width parameter, which is an adjustable parameter in the beam model. In addition, the position of the leaf end can also be modeled using a quadratic formula to account for a shift in the true leaf tip position compared to the nominal position. This adjustable formula, called the MLC position offset (see Section II.B), can be used to model the MLC positioning for machines with MLCs with rounded leaf tips that travel linearly across the field. For the Varian accelerators, this formula mimics the quadratic formula used by the Varian MLC control system to determine the linear distance needed to move the leaves to ensure that the light field edge corresponds to the nominal position. This quadratic formula will depend on the radius of curvature of the leaves. The initial values of the model parameters were determined using vendor recommendations in a process similar to that described by Mzenda et al. [15].

\subsection{MLC leaf offset calibration}

Once a reasonable initial model was created, the MLCX position offset was calibrated. Several methods have been proposed to determine optimal model parameters for the MLC position offset, including comparing films of abutting MLC-defined fields with calculations $[15,16]$ or comparing profiles running parallel to the leaves for fields utilizing a wide range of MLC retracted and over-travel positions $[14,17]$. In this study, we chose to compare profiles acquired at a depth of $10 \mathrm{~cm}$ at $100 \mathrm{~cm}$ SSD to the calculations to optimize the MLCX position offset parameters. The profiles were acquired with a small-volume ion chamber (CCO4, IBA Dosimetry America, Bartlett, TN) and the scans were off-set from the central axis so that the chamber would pass under the center of the MLC leaf. Since it was not possible to import non-symmetric profiles into the treatment planning system, the dose from the planning system was exported and custom Matlab code (Mathworks, Natick, MA) was used to compare the calculations and the measurements.

\subsection{Radial fluence profile}

The off-axis beam profile parameters were initially set to match the calculated and measured inline and crossline profiles through the central axis for the maximum field size, $40 \mathrm{~cm} \times 40 \mathrm{~cm}$. To adjust the beam profile parameters to match the corner of the field, for radial distances approaching $56 \mathrm{~cm}$, the calculated dose for a $40 \mathrm{~cm} \times 40 \mathrm{~cm}$ field was exported and compared using Matlab code to scanned inline and crossline profiles offset $18 \mathrm{~cm}$ from the central axis to run through the corner of the field. Another option is to compare against diagonal scanned profiles [15]. While this procedure optimizes the radial fluence profile to match the dose delivered for large open fields, the dose for smaller fields, centered at highly off-axis positions, may not be as accurate. This effect was discovered during QA of an intensity-modulated radiotherapy (IMRT) plan (created in another planning system) in which a single isocenter was used to treat two separated lesions, resulting in field apertures that were centered significantly off-axis. Despite low modulation in the plan, the IMRT QA failed institutional criteria. To specifically test these conditions during the commissioning process for the current planning system, in addition to the conventional large field profiles, profiles and output measurements were also acquired for $4 \times 4 \mathrm{~cm}$ fields centered at $\pm 16 \mathrm{~cm}$ crossline position and $\pm 8 \mathrm{~cm}$ inline position. Measurements were taken for all 4 positions so that the effect of any beam asymmetry could be taken into account. In Matlab, the calculated dose was compared to the measured profiles and the beam profile parameters adjusted slightly to reduce large disagreements while still maintaining acceptable agreement between measurement and calculation for the large open fields.

\subsection{Flattening filter scatter source}

In RayStation, scatter from the flattening filter is modeled as a Gaussian distribution at an effective distance from the target with an effective width and weight. During initial modeling, the flattening filter width and weight were adjusted to optimize the agreement between the measured and calculated profiles in the out-of-field region, where these parameters have a large impact. Generally, the agreement out-of-field is not as good as that in-field, and the flattening filter parameters represent a compromise between the agreement for small versus large fields and shallow versus deeper depths. During the testing of the model performance, calculated and measured output were compared for small MLC-defined fields with the $X$ and $Y$ jaw position set to $20 \mathrm{~cm} \times 20 \mathrm{~cm}$. Testing the output of small MLC-defined fields with larger jaw positions has been previously described and recommended [5,14,15]. Agreement has generally been described as good once the model had been adjusted using standard techniques. In this study, however, we observed some larger discrepancies for the flattening filter free (FFF) beam models. The addition of a flattening filter weight improved the agreement of the MLC-defined field output, and for these beams the flattening filter parameters were also adjusted to optimize this agreement. Adjusting the flattening filter parameters also required the beam profile parameters to be readjusted and both sets of parameters were iteratively adjusted to optimize agreement of the large field profiles, the small offaxis field output and the output of the small MLC-defined fields with larger jaw opening.

\subsection{MLC parameters}

Initially jaw-defined fields were imported and used for modeling. To perform the initial adjustment of the MLC parameters, the model was copied and the jaw-defined beam data was replaced by MLC-defined beam data. The fields were defined and the profiles scanned such that the inline profiles crossed the edge of the MLC leaf (as opposed to the gap between opposing leaves) and the crossline profiles were slightly offset from the central axis so as to pass under the middle of a MLC leaf (as opposed to the gap between adjacent leaves). As described 
elsewhere, the MLC parameters were also initially checked by comparing film measurements with calculations [15,18]. A number of investigators have noted, however, that using only standard fields or patterns may not lead to optimal MLC parameters for dosimetric accuracy of highly modulated IMRT plans. Several groups have adjusted the MLC parameters away from the values suggested by simple measurements to optimize the agreement between calculations and measurements for delivered IMRT or volumetric-modulated arc therapy (VMAT) plans $[8,16,19]$. Others have utilized special, irregular MLC patterns to find optimal parameters [6,7]. In this study, we utilized IMRT and VMAT plans that had been created using a previously commissioned model in Eclipse version 11 (Varian Medical Systems, Palo Alto, CA). These plans were selected to vary substantially in the irregularity of the MLC patterns, with the most complex spine cases demonstrating MLC patterns involving a high degree of interdigitation. The dose in a low-gradient region was measured using an ion chamber (Pinpoint3D, PTW Freiburg GmbH, Freiburg, Germany) as well as an ArcCHECK diode array (Sun Nuclear Corporation, Melbourne, FL). The MLC parameters, in particular the MLC leaf tip width and to a lesser degree the MLC transmission, were adjusted to optimize the agreement of the calculated dose with the ion chamber measurements over all the plans measured. To check the validity of the modifications made based on ion chamber measurements, a comprehensive set of absolute dose measurements made with a variety of detectors, including diode array and film, were performed as described below.

\subsection{Final model validation}

The final model was validated by comparing calculations to measurements for simple open fields, multiple field configurations, the TG 119 C-shape target test [4], and a battery of clinical IMRT or VMAT plans. For all of the clinical plans, ArcCHECK diode array and ion chamber measurements were performed. The gamma index was used to evaluate the ArcCheck results with a $3 \% / 3 \mathrm{~mm}$ global dose difference and distance-to-agreement criteria and a $10 \%$ threshold dose. A Pinpoint3D ion chamber (PTW Freiburg GmbH, Freiburg, Germany), positioned at the center of the ArcCheck diode array with the cavity plug, was used for absolute dose measurements. For a subset of spine stereotactic body radiotherapy (SBRT) plans, Gafchromic films (Ashland Advanced Materials, Bridgewater, NJ), placed inside of the Stereotactic Dose Verification phantom (Standard Imaging Inc, Middleton, WI), were exposed in an axial plane and analyzed using the SNC Patient software (Sun Nuclear Corporation, Melbourne, FL). The gamma index was used to evaluate the film results with a $3 \% / 3 \mathrm{~mm}$ global dose difference and distance-to-agreement criteria and a $10 \%$ threshold dose.

\section{Results}

\subsection{Initial model adjustment}

The initial model parameters were determined by optimizing the match between the calculations and the measured jaw-defined beam data in the physics module. The percent depth doses (PDDs) generally agreed to within $2 \%$ at depths greater than dmax, and for the most part the agreement was within $1 \%$. In the build-up region, the PDDs generally agreed to within $1 \mathrm{~mm}$ after the first $2 \mathrm{~mm}$ from the surface. The in-field region of the profiles agreed to within $2 \%$. The largest disagreements were observed in the out-of-field region, with maximum discrepancies, expressed as a percentage of the dose on the central axis, as high as $5-6 \%$. As demonstrated in Fig. 1, the computed out-of-field dose tended to be too high for the large fields at shallow depths, but too low for the same field at the deeper depths. As no adjustment could be identified that would adequately change the depth dependence of the out-of-field dose to resolve this issue, the flattening filter and transmission parameters were adjusted to find a reasonable compromise.

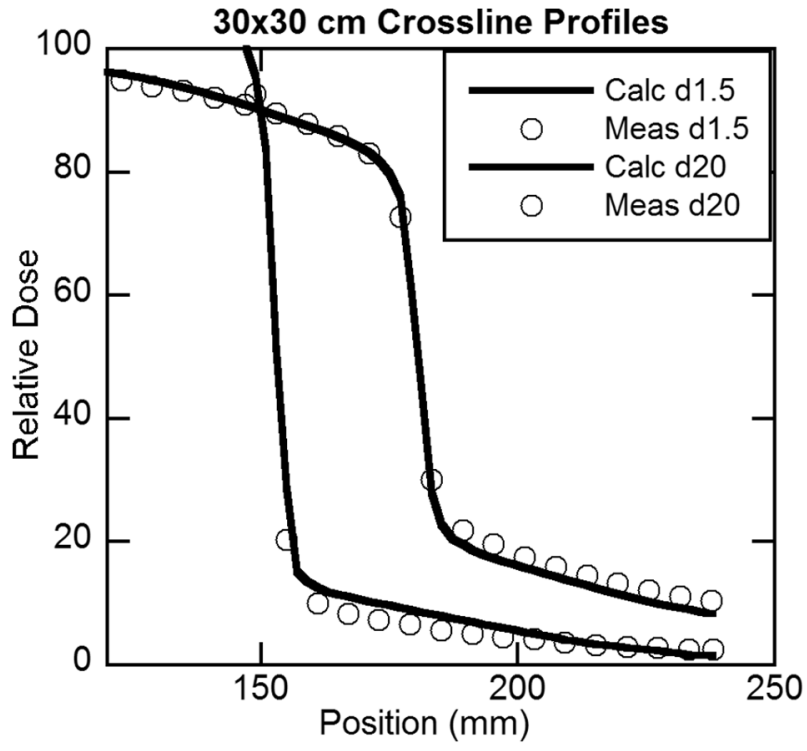

Fig. 1. Comparison of calculated (solid lines) and measured (circles) crossline profiles for a $30 \times 30 \mathrm{~cm}, 6 \mathrm{MV}$ field at $1.5 \mathrm{~cm}$ depth (left curve) and $20 \mathrm{~cm}$ depth (right curve). The calculations over-estimate the dose out-of-field at the shallower depths but under-estimate the dose out-of-field at deeper depths.

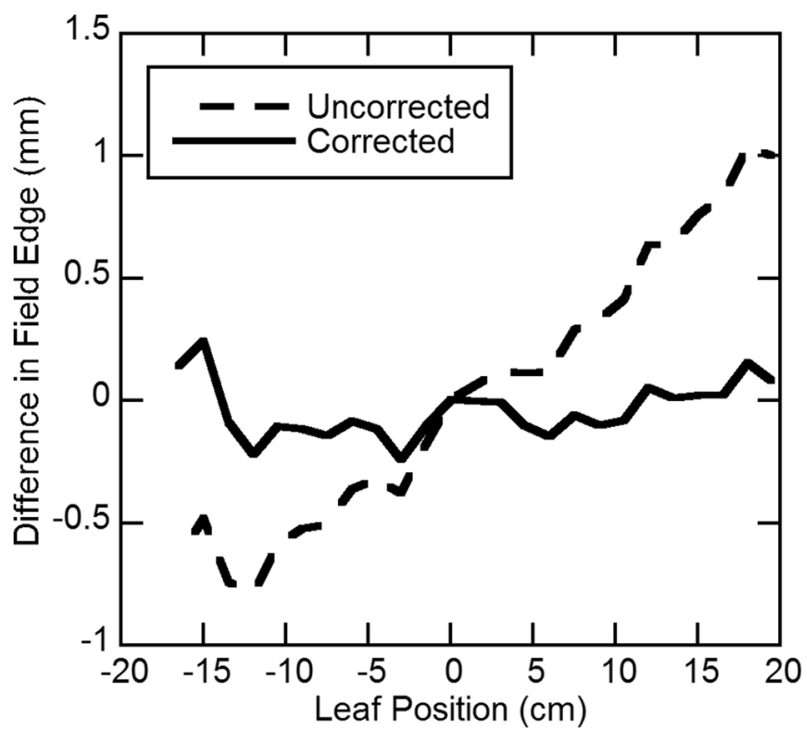

Fig. 2. Difference in the calculated versus measured field edge for crossline profiles through the MLCs as a function of the leaf position.

\subsection{MLC offset adjustment}

The impact of adjusting the MLCX calibration is demonstrated in Fig. 2. Without any calibration, there is a small but systematic deviation of the calculated MLC field edges compared to the measured field edges. The RayStation system allows the offset of the true MLC leap tip position from the nominal position to be modeled as a quadratic function of leaf position. As illustrated in Fig. 2, the offset in this case is fairly linear and a linear fit was performed to the data and input into the MLC X calibration parameters. With this correction, the deviation between the calculated and measured field edges is less than $0.5 \mathrm{~mm}$ at all positions spanning from $16 \mathrm{~cm}$ over-travelled to $19 \mathrm{~cm}$ retracted.

\subsection{Off-axis beam profile adjustment}

The importance of using profiles running through the corner of the 
a

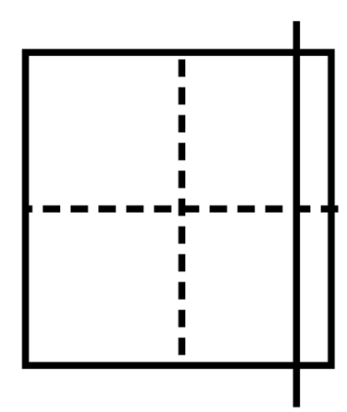

b

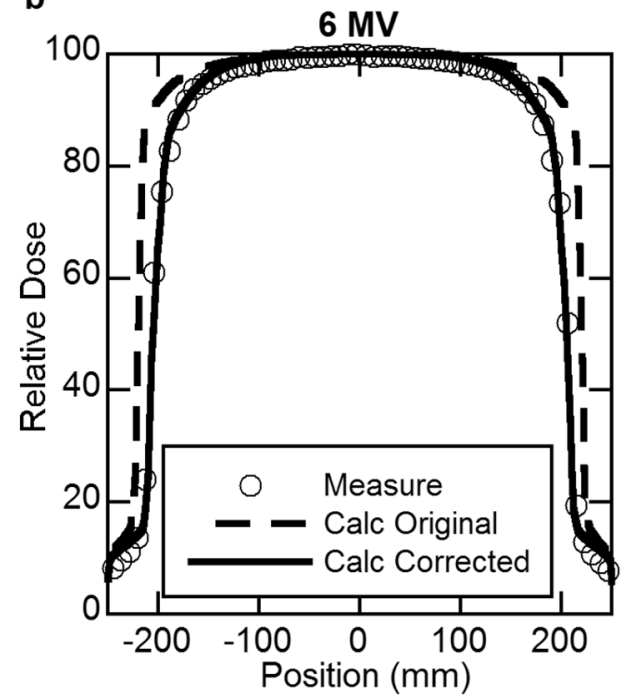

C

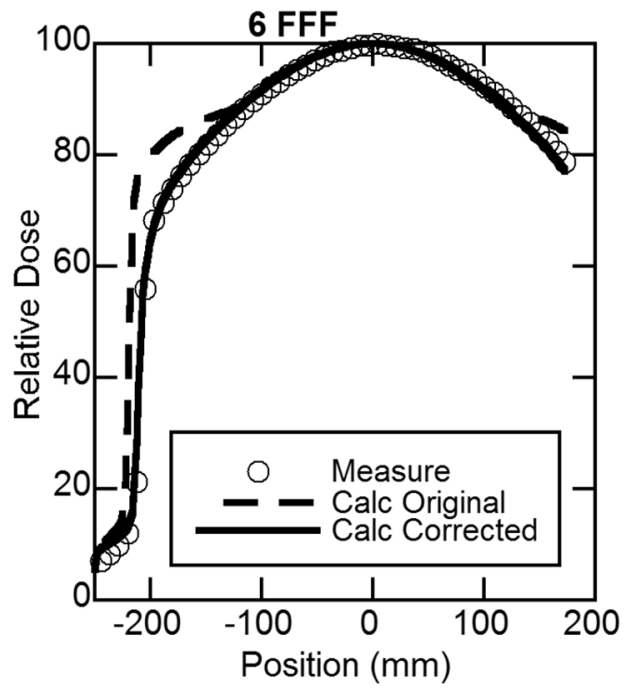

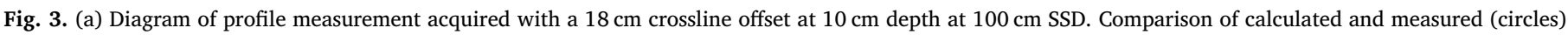

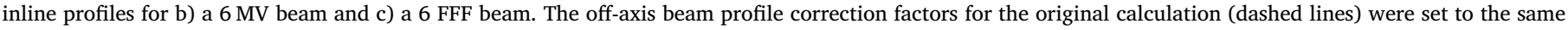

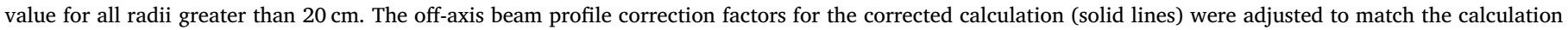
with the measurements.

a

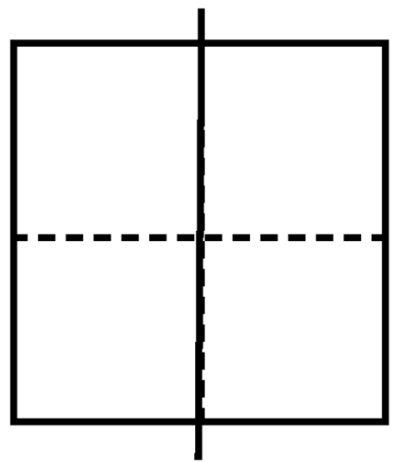

C

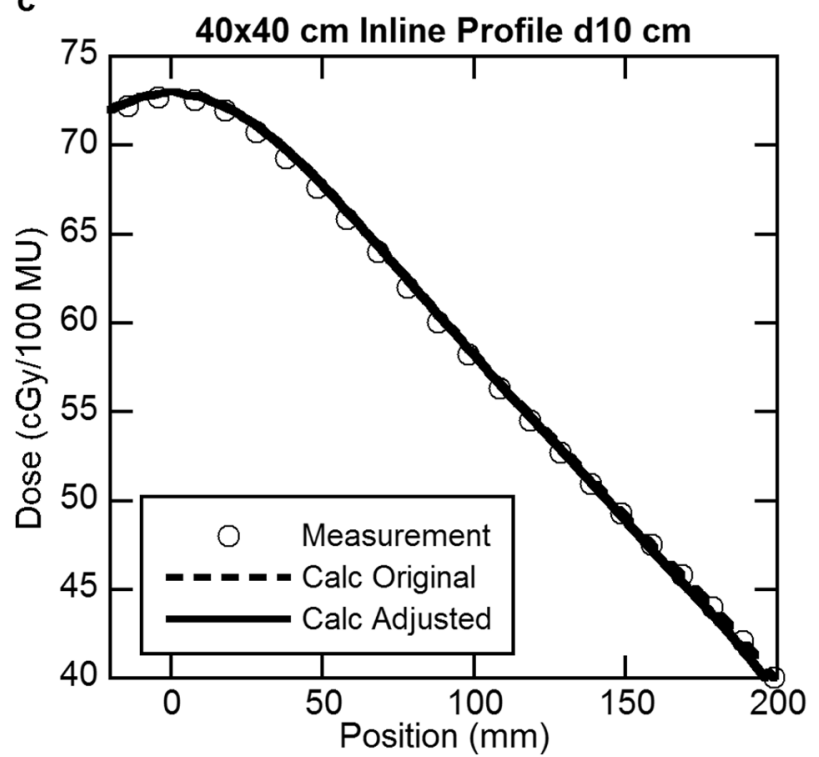

b

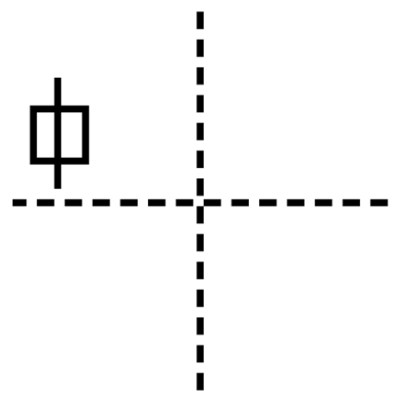

d

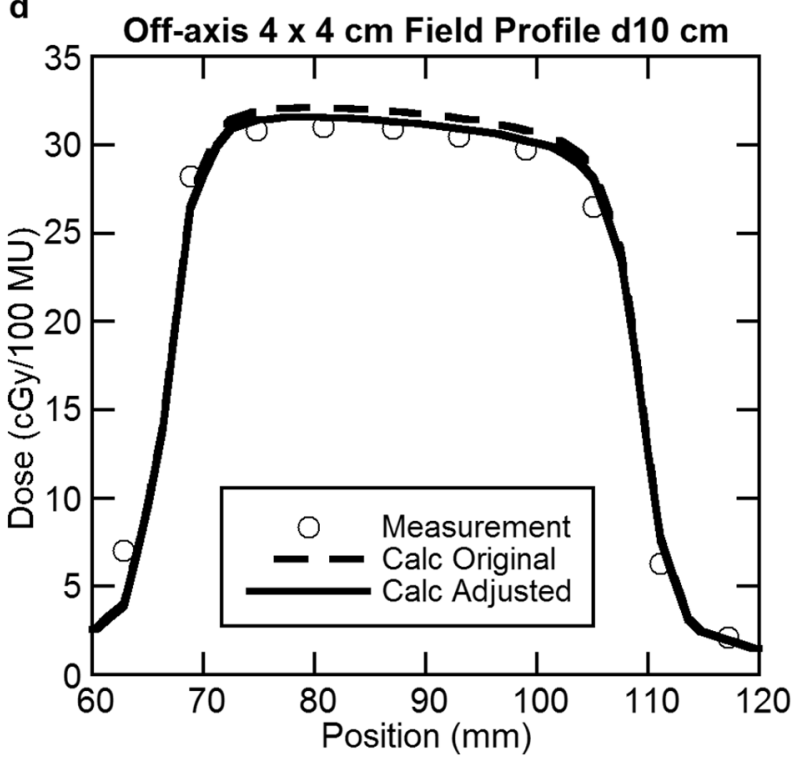

Fig. 4. Comparison of measured profiles (circles), calculated profiles for the original model parameters (dashed line) and calculated profiles with the adjusted model parameters (solid line) for the 6FFF beam model. On the left, (a) measurement diagram and (c) inline profiles for a $40 \times 40 \mathrm{~cm}$ field at $10 \mathrm{~cm}$ depth. On the right, (b) measurement diagram and (d) inline profiles at $10 \mathrm{~cm}$ depth running through the middle of a $4 \times 4 \mathrm{~cm}$ field that is centered $16 \mathrm{~cm}$ crossline and $8 \mathrm{~cm}$ inline offset from the central axis. 
Table 1

Effect of flattening filter weight on calculated dose for MLC-defined fields.

\begin{tabular}{lllll}
\hline & $\begin{array}{l}6 \mathrm{FFF} \\
\text { original }\end{array}$ & $\begin{array}{l}6 \mathrm{FFF} \\
\text { adjusted }\end{array}$ & $\begin{array}{l}10 \mathrm{FFF} \\
\text { original }\end{array}$ & $\begin{array}{l}10 \mathrm{FFF} \\
\text { adjusted }\end{array}$ \\
\hline $\begin{array}{l}\text { Model parameters } \\
\text { Flattening filter }\end{array}$ & 0.000 & 0.065 & 0.000 & 0.040 \\
$\quad$ weight & & & \\
$\quad$ Difference between calculated and measured doses for & MLC-defined fields with $20 \times 20 \mathrm{~cm}$ \\
$\quad$ jaw positions & & & & \\
$3 \times 3 \mathrm{~cm}$ MLC & $4.1 \%$ & $0.1 \%$ & $2.6 \%$ & $0.2 \%$ \\
$5 \times 5 \mathrm{~cm}$ MLC & $3.8 \%$ & $-0.3 \%$ & $2.0 \%$ & $-0.4 \%$ \\
$10 \times 10 \mathrm{~cm}$ MLC & $2.0 \%$ & $1.4 \%$ & $1.3 \%$ & $0.8 \%$ \\
$15 \times 15 \mathrm{~cm}$ MLC & $0.9 \%$ & $0.6 \%$ & $1.0 \%$ & $0.3 \%$ \\
\hline
\end{tabular}

* Note: The beam profiles were also adjusted when the flattening filter weight was changed.

Table 2

Effect of MLC leaf tip width and transmission on calculated dose for VMAT plans.

\begin{tabular}{lllll}
\hline & $\begin{array}{l}6 \mathrm{MV} \\
\text { original }\end{array}$ & $6 \mathrm{MV}$ final & $\begin{array}{l}6 \mathrm{FFF} \\
\text { original }\end{array}$ & $6 \mathrm{FFF}$ final \\
\hline Model parameters & & & & \\
MLC leaf tip width & $0.18 \mathrm{~cm}$ & $0.45 \mathrm{~cm}$ & $0.20 \mathrm{~cm}$ & $0.40 \mathrm{~cm}$ \\
MLC transmission & 0.0146 & 0.010 & 0.0146 & 0.008 \\
Difference between calculated and measured doses & & \\
GBM - High dose & $-2.0 \%$ & $-1.0 \%$ & $-0.5 \%$ & $-1.0 \%$ \\
Simple spine - High dose & $0.0 \%$ & $0.9 \%$ & $1.2 \%$ & $1.7 \%$ \\
Simple spine - Cord & $-1.6 \%$ & $0.3 \%$ & $-2.0 \%$ & $-1.8 \%$ \\
Complex spine - High & $-1.7 \%$ & $2.1 \%$ & $-0.8 \%$ & $0.8 \%$ \\
$\quad$ dose & $-12.8 \%$ & $-2.3 \%$ & $-6.5 \%$ & $-0.7 \%$ \\
Complex spine - Cord & & & & \\
\hline
\end{tabular}

Table 3

Agreement between calculations and point measurements for single fields.

\begin{tabular}{ll}
\hline Conditions & $\begin{array}{l}\text { Agreement between calculation and } \\
\text { measurement }\end{array}$ \\
\hline $\begin{array}{l}\text { Center of open jaw or MLC-defined } \\
\text { field }\end{array}$ & Within $2 \%$ \\
$\begin{array}{l}\text { Off-axis point in open field } \\
\text { Center of asymmetric open field }\end{array}$ & Within $1 \%$ \\
& Mostly within $2.5 \%$. 6FFF 3.8\% for pt \\
Rectangular field & 18 cm off-axis \\
$80 / 120$ cm SSD & Within $1 \%$ \\
Mantle field & Within $\sim 1.5 \%$ \\
& Open region: Within $1 \%$ \\
Oblique incidence and Flash & Shielded region: Up to $10 \%$ disagreement \\
$1 \mathrm{~cm}$ beyond Lung slab & Within $1 \%$ \\
$2.5 \mathrm{~cm}$ beyond Bone slab & Mostly within $2.5 \%$. 6 FFF: $4.1 \%$ \\
$2.5 \mathrm{~cm}$ beyond Air cavity & Within $2 \%$ \\
& Mostly within $2 \% .6$ FFF: $3.3 \%$
\end{tabular}

largest field size for adjusting the off-axis beam profile parameters is highlighted in Fig. 3. In the figure, TPS calculations are compared to measured inline profiles acquired with an $18 \mathrm{~cm}$ crossline offset at $10 \mathrm{~cm}$ depth, $100 \mathrm{~cm}$ SSD. The off-axis beam profile correction factors for the original calculation were set to the same value for all radii greater than $20 \mathrm{~cm}$, and subsequently adjusted to match the calculation with the measurements for the profiles with the large offset away from the central axis. Both models produce the same values for profiles through the central axis of a $40 \times 40 \mathrm{~cm}$ field. In particular for the FFF beam, the off-axis fluence is considerably over-estimated if the beam profile correction factors for radii greater than $20 \mathrm{~cm}$ are not manually adjusted to reflect the measured dose in the corner of the $40 \times 40 \mathrm{~cm}$ field.

Fig. 4 illustrates how the beam profile at large off-axis distances was adjusted as a compromise between the agreement for an open $40 \times 40 \mathrm{~cm}$ field and for small fields centered at highly off-axis positions. In this example, the 6FFF beam model was initially adjusted to match the $40 \times 40 \mathrm{~cm}$ field measured beam profiles to within $0.5 \%$ (Fig. 4 left - dashed line). Using this model, however, the calculated dose for a $4 \times 4 \mathrm{~cm}$ field offset $16 \mathrm{~cm}$ crossline and $8 \mathrm{~cm}$ inline from the central axis was over $3 \%$ different from the measured dose (Fig. 4 right - dashed line). The beam profile parameter was adjusted so that for both configurations, the calculations were within $2 \%$ of the measured doses (Fig. 4 - solid lines).

\subsection{Flattening filter scatter source}

Table 1 demonstrates the effect of adding a flattening filter weight on the accuracy of the dose calculated for MLC-defined fields with wider jaw settings for FFF beam models. Because there is no physical flattening filter in the beamline, the flattening filter weight was initially set to 0.000 . As seen in Table 1 , by adding a non-zero flattening filter weight (and adjusting the beam profile as needed to match the large field and off-set field profiles) the calculated MLC-defined field output was improved to within $2 \%$ accuracy, compared to a maximum difference of $4 \%$ for the original beam models.

\subsection{MLC parameters}

The final stage of beam adjustment was to tune the MLC parameters to maximize the agreement of the measured and calculated doses for VMAT plans of varying complexity. The initial MLC parameters were set by adjusting the parameters to best match the calculated profiles with measured profiles for static open MLC-defined fields. As seen in Table 2, for the more simple plans (GBM, simple spine) the calculated doses agree well with the ion chamber measurements using the initial parameters. However, the calculated dose in the cord region of the highly modulated spine VMAT plan disagreed with the measured dose by $12 \%$ for the initial $6 \mathrm{MV}$ model and by $6 \%$ for the initial $6 \mathrm{FFF}$ model. After adjusting the MLC parameters to optimize the agreement for all cases, the calculated doses were all within $2.5 \%$ of the measurement. Notably, the optimized MLC leaf tip width was more than twice the original value.

\subsection{Final model validation}

The final model was first validated by comparing the calculated and measured doses for single fields of various jaw/MLC positions, different depths, and SSD. Calculations were also compared for oblique incidence and beyond different inhomogeneous materials. As summarized in

Table 4

Comparison of calculations and measurements for various clinical cases.

\begin{tabular}{|c|c|c|c|c|c|}
\hline \multirow[t]{2}{*}{ Case } & \multicolumn{5}{|c|}{ Ion chamber agreement (\%)/ArcCHECK passing rate (\%) for $3 \% / 3 \mathrm{~mm}$ criteria } \\
\hline & $6 \mathrm{MV}$ & $10 \mathrm{MV}$ & $15 \mathrm{MV}$ & $6 \mathrm{FFF}$ & $10 \mathrm{FFF}$ \\
\hline Anal/Rectal & $0.0 / 99.4$ & $-1.0 / 100.0$ & $0.6 / 99.9$ & $-1.8 / 98.2$ & $-2.0 / 97.1$ \\
\hline Head-Neck & $-0.2 / 99.1$ & $-1.4 / 99.9$ & $-1.5 / 99.9$ & NA & NA \\
\hline Simple Spine & $1.8 / 100.0$ & $-1.7 / 97.0$ & $2.5 / 97.8$ & $-2.2 / 97.0$ & $-2.0 / 97.6$ \\
\hline Complex Spine & $-1.1 / 98.5$ & $-1.7 / 97.6$ & $-2.9 / 98.5$ & $-2.4 / 96.8$ & $-4.1 / 96.2$ \\
\hline
\end{tabular}




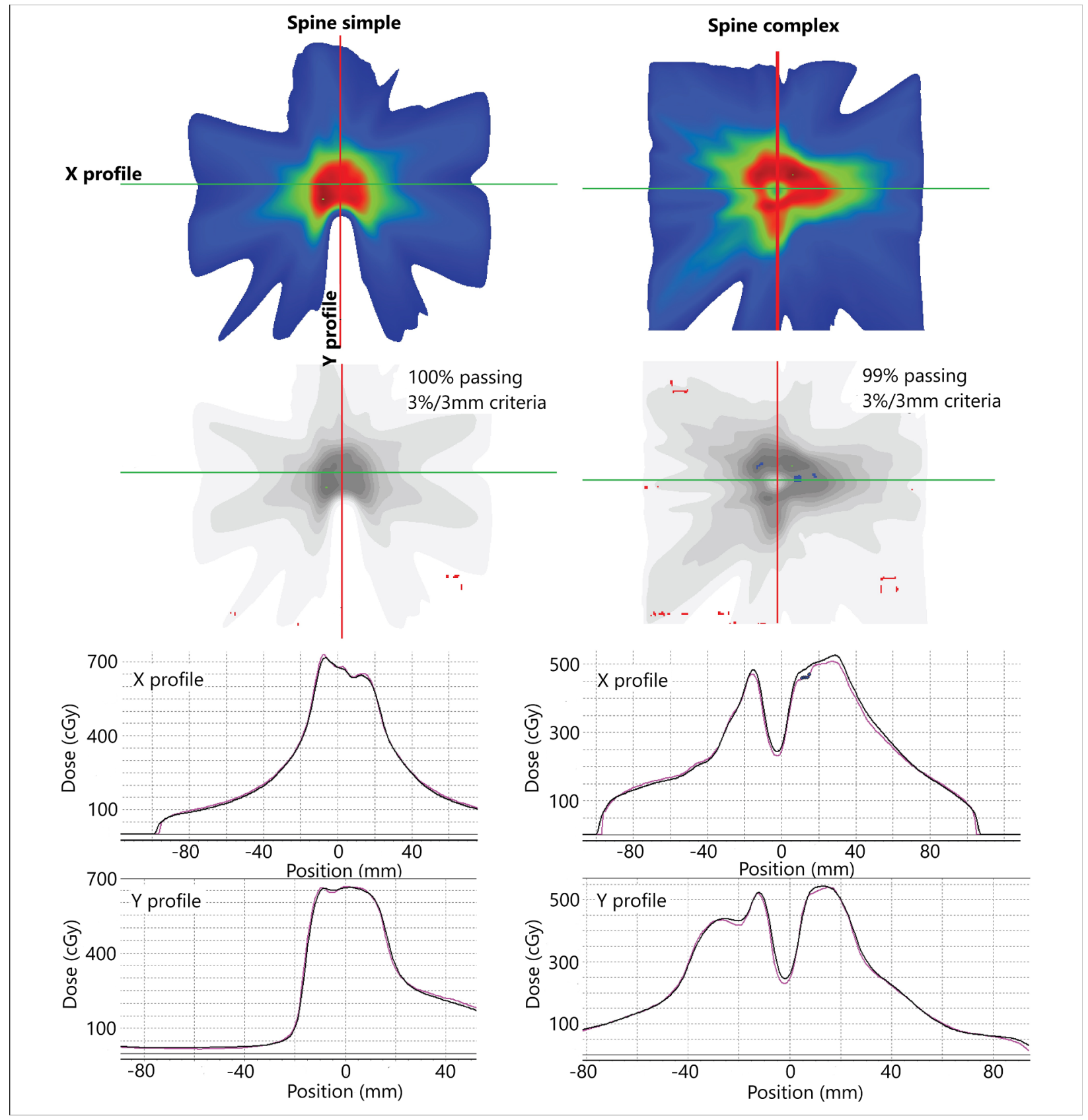

Fig. 5. Comparison between film measurements and calculations for simple and complex spine cases.

Table 3, in general, agreement was within $2 \%$. The TG $119 \mathrm{C}$ shape target was planned for segmental MLC (SMLC), dynamic MLC (DMLC), and VMAT techniques for all energies, and the calculated dose agreed to the measured dose to within $2 \%$ of the prescribed dose for both the target and cord regions. A variety of clinical cases, ranging from a large anal/rectal plan to small spine lesions, were planned in RayStation and measured with an ion chamber and the ArcCHECK diode device; results are presented in Table 4 . In general the ion chamber agreement was within $3 \%$, and the passing rates above $95 \%$ using a $3 \% / 3 \mathrm{~mm}$ gamma criteria. The beam model with the greatest deviation between the calculated and measured doses for the clinical IMRT/VMAT cases was the 10 FFF model. It may be that the 10 FFF IMRT plans tend to be more modulated than the plans for the other beam energies, as additional modulation is needed to compensate for the highly peaked profile of the beam. However the agreement for the 10 FFF model was still overall within acceptable tolerances. Fig. 5 demonstrates the excellent agreement that was also observed comparing film measurements with calculations for the spine cases. Based on these results, our institution accepted the model for clinical use for all cases with the exception of stereotactic radiotherapy of multiple brain lesions. The extensive use of the MLCs to shield the regions between separated targets in these types of plans warrants further specific testing of the dosimetric accuracy in the non-target region that has not been part of this process.

\section{Discussion}

As demonstrated by the recent IROC-Houston study [9], commissioning an external beam treatment planning system for dosimetric accuracy continues to be a major challenge for the radiation oncology community. As the delivery techniques have become more sophisticated, such as with the introduction of VMAT, the difficulty of the task has increased. The recent medical physics practice guideline on 
commissioning of treatment planning dose calculations [5] emphasizes the importance of testing the dosimetric accuracy of the TPS under a variety of conditions. This study provides a practical example and procedure for how a model can be adjusted and improved through this systematic process.

One surprising observation in this study was the effect of the flattening filter (non-point-like) source model on the output for MLC-defined fields when the jaws are opened wider than the MLCs. To our knowledge, this effect has not been documented previously. Adding a flattening filter component to the FFF beam model improved the agreement between calculations and measurements for these MLC-defined fields, from a maximum difference of $4 \%$ to within $2 \%$ agreement. Others have also reported using a non-zero scattering source for FFF beam models $[20,21]$. A thin brass foil is used in place of the flattening filter during the FFF treatment mode on the TrueBeam. This foil as well as multiple scattering of photons in other collimating and shielding elements in the head may contribute to a non-point-like source of radiation for the FFF beams.

This study also reinforces the need of incorporating highly irregular MLC-defined fields and IMRT plans as part of the adjustment of the MLC model parameters. Numerous other studies have also demonstrated that making simple MLC transmission or gap measurements does not guarantee good performance for clinical IMRT plans $[6,7,8,16]$. In this study, differences of over $10 \%$ between calculation and measurement were found when the MLC parameters were adjusted only using profiles for static/regularly-shaped MLC fields. After adjusting the MLC parameters, the calculations agreed to within $3 \%$ for the test plans as well as for a battery of clinical validation cases. Another option to comparing the planning system calculations to direct measurements is to compare the calculation to a well-validated Monte Carlo simulation calculation as was performed by Onizuka et al. [22]. The advantage of comparing against Monte Carlo simulation is that the full three-dimensional dose distribution can be compared as opposed to point or two-dimensional measured distributions. However, the Monte Carlo simulation itself would first need to be appropriately validated against measurements.

\section{Conclusions}

This study has demonstrated a systematic approach to optimizing beam model parameters for dosimetric accuracy over a wide range of treatment conditions. The process detected potential dosimetric errors of over $10 \%$ and resulted in a model that provided in general $3 \%$ dosimetric accuracy.

\section{Funding}

This research did not receive any specific grant from funding agencies in the public, commercial or not-for-profit sectors.

\section{Declaration of interest}

Several authors participate in a collaboration agreement with RaySearch Laboratories. This work, however, was part of our clinical commissioning process and was independent of the collaboration.

\section{References}

[1] Fraass B, Doppke K, Hunt M, Kutcher G, Starkschall G, Stern R, et al. American association of physicists in medicine radiation therapy committee task group 53: quality assurance for clinical radiotherapy treatment planning. Med Phys 1998;25(10):1773-829.

[2] Ezzell GA, Galvin JM, Low D, Palta JR, Rosen I, Sharpe MB, et al. Guidance document on delivery, treatment planning, and clinical implementation of IMRT: report of the IMRT subcommittee of the AAPM radiation therapy committee. Med Phys 2003;30(8):2089-115.

[3] International Atomic Energy Agency. Commissioning and quality assurance of computerized planning systems for radiation treatment of cancer. TRS 430. Vienna: International Atomic Energy Agency; 2004. p. 67.

[4] Ezzell GA, Burmeister JW, Dogan N, LoSasso TJ, Mechalakos JG, Mihailidis D, et al IMRT commissioning: multiple institution planning and dosimetry comparisons, a report from AAPM Task Group 119. Med Phys 2009;36(11):5359-73.

[5] Smilowitz JB, Das IJ, Feygelman V, Fraass BA, Kry SF, Marshall IR, et al. Medical physics practice guideline 5.a.: commissioning and QA of treatment planning dose calculations - Megavoltage photon and electron beams. J Appl Clin Med Phys 2015;16(5):14-34.

[6] Letourneau D, Sharpe MB, Owrangi A, Jaffray DA. Automated beam model optimization. Med Phys 2010;37(5):2110-20.

[7] Yao W, Farr JB. Determining the optimal dosimetric leaf gap setting for rounded leaf-end multileaf collimator systems by simple test fields. J Appl Clin Med Phys 2015;16(4):65-77.

[8] Szpala S, Cao F, Kohli K. On using the dosimetric leaf gap to model the rounded leaf ends in VMAT/RapidArc plans. J Appl Clin Med Phys 2014;15(2):67-84.

[9] Kerns JR, Stingo F, Followill DS, Howell RM, Melancon A, Kry SF. Treatment planning system calculation errors are present in most Imaging and Radiation Oncology Core-Houston phantom failures. Int J Radiat Oncol Biol Phys 2017;98(5):1197-203.

[10] Das IJ, Cheng C-W, Watts RJ, Ahnesjo A, Gibbons J, Li XA, et al. Accelerator beam data commissioning equipment and procedures: report of the TG-106 of the therapy physics committee of the AAPM. Med Phys 2008;35(9):4186-215.

[11] RaySearch Laboratories AB. RayStation 5 reference manual. Stockholm, Sweden: RaySearch Laboratories AB; 2016. p. 250.

[12] Mackie TR, Scrimger JW, Battista JJ. A convolution method of calculating dose for 15-MV xrays. Med Phys 1985;12(2):188-96.

[13] Ahnesjo A. Collapsed cone convolution of radiant energy for photon dose calculation in heterogeneous media. Med Phys 1989;16(4):577-92.

[14] Chen S, Yi BY, Yang X, Xu H, Prado KL, D'Souza WD. Optimizing the MLC model parameters for IMRT in the RayStation treatment planning system. J Appl Clin Med Phys 2015;16(5):322-32.

[15] Mzenda B, Mugabe KV, Sims R, Godwin G, Loria D. Modeling and dosimetric performance evaluation of the RayStation treatment planning system. J Appl Clin Med Phys 2014;15(5):29-46.

[16] Kielar KN, Mok E, Hsu A, Wang L, Luxton G. Verification of dosimetric accuracy on the TrueBeam STx: rounded leaf effect of the high definition MLC. Med Phys 2012;39(10):6360-71.

[17] Rice JR. Optimization of the rounded leaf offset table in modeling the multileaf collimator leaf edge in a commercial treatment planning system. J Appl Clin Med Phys 2014;15(6):128-37.

[18] Cadman P, McNutt T, Bzdusek K. Validation of physics improvements for IMRT with a commercial treatment-planning system. J Appl Clin Med Phys 2005;6(2):74-86.

[19] Wen N, Zhao B, Kim J, Chin-Snyder K, Bellon M, Glide-Hurst C, et al. IMRT and RapidArc commissioning of a TrueBeam linear accelerator using TG-119 protocol cases. J Appl Clin Med Phys 2014;15(5):74-88.

[20] Foster RD, Speiser MP, Solberg TD. Commissioning and verification of the collapsed cone convolution superposition algorithm for SBRT delivery using flattening filterfree beams. J Appl Clin Med Phys 2014;15(2):39-49.

[21] Valdenaire S, Mailleux H, Fau P. Modeling of flattening filter free photon beams with analytical and Monte Carlo TPS. Biomed Phys Eng Express 2016;2:035010.

[22] Onizuka R, Araki F, Ohno T. Monte Carlo dose verification of VMAT plans using Elekta Agility 160-leaf MLC. Phys Medica 2018;51:22-31. 